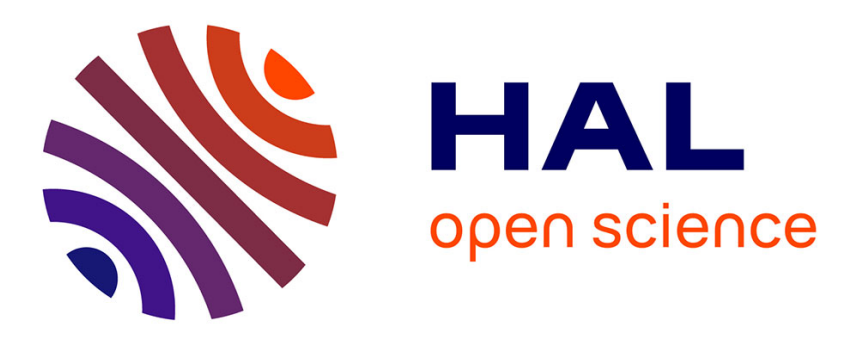

\title{
Fault tolerant tracking control for continuous Takagi-Sugeno systems with time varying faults
}

Tahar Bouarar, Benoît Marx, Didier Maquin, José Ragot

\section{To cite this version:}

Tahar Bouarar, Benoît Marx, Didier Maquin, José Ragot. Fault tolerant tracking control for continuous Takagi-Sugeno systems with time varying faults. 19th Mediterranean Conference on Control and Automation, MED'11, Jun 2011, Corfou, Greece. pp.1106-1111, 10.1109/MED.2011.5983160 . hal-00593396

\section{HAL Id: hal-00593396 https://hal.science/hal-00593396}

Submitted on 8 Apr 2014

HAL is a multi-disciplinary open access archive for the deposit and dissemination of scientific research documents, whether they are published or not. The documents may come from teaching and research institutions in France or abroad, or from public or private research centers.
L'archive ouverte pluridisciplinaire HAL, est destinée au dépôt et à la diffusion de documents scientifiques de niveau recherche, publiés ou non, émanant des établissements d'enseignement et de recherche français ou étrangers, des laboratoires publics ou privés. 


\title{
Fault tolerant tracking control for continuous Takagi-Sugeno systems with time varying faults
}

\author{
Tahar Bouarar, Benoît Marx, Didier Maquin and José Ragot
}

\begin{abstract}
This paper deals with Fault Tolerant Control design for continuous nonlinear Takagi-Sugeno faulty systems. The goal is to ensure both state and fault estimation and the state reference tracking even if faults occur. In this study, the faults affecting the system behavior are considered as time varying functions modeled by exponential functions or first order polynomials. Based on descriptor redundancy property, solutions are proposed for both cases, exponential and polynomial faults, in terms of Linear Matrix Inequalities (LMI). In order to illustrate the applicability and the effectiveness of the proposed approaches, numerical examples are considered.
\end{abstract}

\section{INTRODUCTION}

The modern control of physical systems uses new algorithm approaches in order to increase the performances and the safety requirements. The classical control methodologies for nonlinear complex systems can lead to unsatisfactory performances, or even in some cases, to the instability if any malfunction occurs in the process components (as sensors or actuators). To overcome these drawbacks, new control system techniques have been developed in order to guarantee the overall system stability and acceptable performances, despite the failure situation. This new strategy of system control is called Fault Tolerant Control (FTC). The main idea of this strategy relies on the adaptation of the control law on the basis of the estimation of the faults affecting the system. Many works dealing with FTC design have been developed. For example, in the linear framework, the FTC problem is widely treated [1][2][3][4][5][6][7][8]. However in practice, most of physical systems are nonlinear, hence, it is primary to consider the FTC design for nonlinear systems. Some approaches dealing with this problem are proposed by [9][10][11]. Nevertheless, these approaches are applied only when the operating points change or the fault occurs. Thus, the approaches taking into account the changes caused by both operating point variations and fault are not yet available (for more details, the reader can refer to [7]). Indeed, a way to overcome the existing FTC approach drawbacks is to consider the nonlinear Takagi-Sugeno models [12]. This class of models allows representing exactly the overall nonlinear systems on its operating region in the state space. TakagiSugeno models are described by a set of Linear Time Invariant (LTI) models, each LTI model represents the nonlinear system behavior in a particular region in the state space. The

\footnotetext{
This work was supported by the GIS 3SGS

All the authors are with Centre de Recherche en Automatique de Nancy (CRAN) UMR 7039. Nancy-Université, CNRS, 2, avenue de la forêt de Haye, 54516 Vandoeuvre-Lès-Nancy, France. \{tahar.bouarar, benoit.marx, didier.maquin, jose.ragot, \}@ensem.inpl-nancy.fr
}

overall nonlinear system dynamics is obtained by blending together all the LTI models with nonlinear functions. Since introducing Takagi-Sugeno models, many works dealing with stability, stabilization and estimation have been developed $[13][14][15][16]$. Nevertheless, the FTC design problem has been few treated. For example, the trajectory tracking FTC design for Takagi-Sugeno model subject to actuator constant fault has been developed by [17], when the premise variables are measurable and by [18][19] when they are unmeasurable. In these studies, the estimation of the constant faults is obtained by using proportional integral observers. Many papers reported that, on a practical point of view, even if the considered faults are not constant but only slow varying (with regards the dynamics of the system), the proposed observers provide good results.

However, in this paper, our goal is to study the problem of FTC design for Takagi-Sugeno models subject to time varying faults. The idea is to take into account an a priori knowledge about the faults acting on the system and investigate how the fault estimation can take advantage of that. Two particular fault models are considered: exponential function and first order polynomial. Thus, two approaches are proposed to ensure the state tracking between the healthy system and the faulty ones.

The following notations are considered to improve the paper readability: $X_{\mu}=\sum_{i=1}^{r} \mu(\xi(t)) X_{i}$. In a block matrix, a star, $*$, denotes the transposed element in the symmetric position and $\operatorname{diag}\left(\Theta_{1}, \ldots, \Theta_{r}\right)$ is a block diagonal matrix which diagonal entries are defined by $\Theta_{1}, \ldots, \Theta_{r}$. The following lemmas are needed to provide LMI conditions.

Lemma 1 [20]: Consider two real matrices $\Pi$ and $Z$ with appropriate dimensions, for any positive scalar $\tau$ the following inequality holds:

$$
\Pi^{T} \mathrm{Z}+\mathrm{Z}^{T} \Pi \leq \tau \Pi^{T} \Pi+\tau^{-1} \mathrm{Z}^{T} \mathrm{Z}
$$

Lemma 2 [24]: Consider the matrices $\mathrm{T}_{i}=\mathrm{T}_{i}^{T}, i \in\{0, \ldots, k\}$. The following expressions are equivalent:

$$
\begin{gathered}
\forall \zeta, \zeta^{T} \mathrm{~T}_{0} \zeta \geq 0 \text { and } \zeta^{T} \mathrm{~T}_{i} \zeta \geq 0, \forall i \in\{1, \ldots, k\} \\
\exists \rho_{1} \geq 0, \ldots, \rho_{k} \geq 0 \text { such that } \forall \zeta, \mathrm{T}_{0}-\sum_{i=1}^{k} \rho_{i} \mathrm{~T}_{i} \geq 0
\end{gathered}
$$




\section{PROBLEM FORMULATION}

Let us consider the following reference model:

$$
\left\{\begin{array}{l}
\dot{x}(t)=\sum_{i=1}^{r} \mu_{i}(\xi(t))\left(A_{i} x(t)+B_{i} u(t)\right) \\
y(t)=\sum_{i=1}^{r} \mu_{i}(\xi(t))\left(C_{i} x(t)+D_{i} u(t)\right)
\end{array}\right.
$$

where $x(t) \in \mathbb{R}^{n}, y(t) \in \mathbb{R}^{p}$ and $u(t) \in \mathbb{R}^{m}$ are respectively the state, the output and the nominal control vectors. The $r$ submodels are defined by the matrices $A_{i} \in \mathbb{R}^{n \times n}, B_{i} \in$ $\mathbb{R}^{n \times m}, C_{i} \in \mathbb{R}^{p \times n}$ and $D_{i} \in \mathbb{R}^{p \times m} . \mu_{i}(\xi(t))$ are the nonlinear functions depending on the variable $\xi(t)$ which can be measurable $(u(t)$ or $y(t))$ or unmeasurable $(x(t))$. These nonlinear functions satisfy the convex sum property: $\forall t$

$$
\left\{\begin{array}{l}
0 \leq \mu_{i}(\xi(t)) \leq 1, \quad i=1, \ldots, r \\
\sum_{i=1}^{r} \mu_{i}(\xi(t))=1
\end{array}\right.
$$

Let us consider the following faulty system:

$$
\left\{\begin{array}{l}
\dot{x}_{f}(t)=\sum_{i=1}^{r} \mu_{i}(\xi(t))\left(A_{i} x_{f}(t)+B_{i} u_{f}(t)+G_{i} f(t)\right) \\
y_{f}(t)=\sum_{i=1}^{r} \mu_{i}(\xi(t))\left(C_{i} x_{f}(t)+D_{i} u_{f}(t)+W_{i} f(t)\right)
\end{array}\right.
$$

where $x_{f}(t) \in \mathbb{R}^{n}, y_{f}(t) \in \mathbb{R}^{p}, f(t) \in \mathbb{R}^{q}$ and $u_{f}(t) \in \mathbb{R}^{m}$ are respectively the faulty state, the faulty output, the fault affecting the system and the fault tolerant control vectors. $G_{i} \in \mathbb{R}^{n \times q}$ and $W_{i} \in \mathbb{R}^{p \times q}$ are the transfer matrices of the faults on the system. The fault tolerant controller design methodology is based on the following scheme.

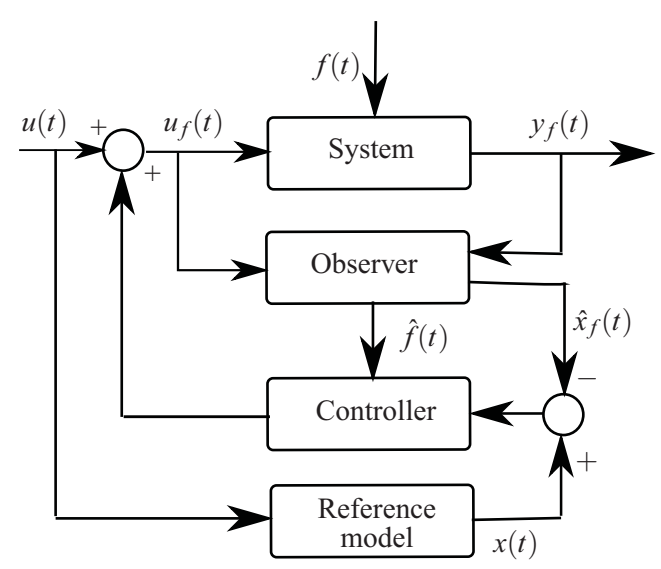

Fig. 1. Tracking fault tolerant controller design scheme

In order to ensure the tracking between the healthy system states and the faulty system ones, we consider the following FTC law given by:

$$
u_{f}(t)=u(t)+\sum_{i=1}^{r} \mu_{i}(\xi(t))\left(K_{i}\left(x(t)-\hat{x}_{f}(t)\right)-K_{i}^{f} \hat{f}(t)\right)
$$

where $K_{i} \in \mathbb{R}^{m \times n}$ and $K_{i}^{f} \in \mathbb{R}^{m \times q}$ are the state feedback gain matrices to be determined. In this study, an observer is needed to estimate simultaneously the faults and the faulty system states. The observer structure is given by:

$$
\left\{\begin{array}{l}
\dot{\hat{x}}_{f}(t)=\sum_{i=1}^{r} \mu_{i}(\xi(t))\left(A_{i} \hat{x}_{f}(t)+B_{i} u_{f}(t)+G_{i} \hat{f}(t)+\varphi_{i}(t)\right) \\
\hat{y}_{f}(t)=\sum_{i=1}^{r} \mu_{i}(\xi(t))\left(C_{i} \hat{x}_{f}(t)+D_{i} u_{f}(t)+W_{i} \hat{f}(t)\right) \\
\dot{\hat{f}}(t)=\sum_{i=1}^{r} \mu_{i}(\xi(t))\left(H_{i}^{2}\left(y_{f}(t)-\hat{y}_{f}(t)\right)-H_{i}^{3} \hat{f}(t)\right)
\end{array}\right.
$$

with $\varphi_{i}(t)=H_{i}^{1}\left(y_{f}(t)-\hat{y}_{f}(t)\right)$. The observer's gain matrices $H_{i}^{1} \in \mathbb{R}^{n \times p}, H_{i}^{2} \in \mathbb{R}^{q \times p}$ and $H_{i}^{3} \in \mathbb{R}^{q \times q}$ are to be designed. With this FT controller structure, one can remark that fault detection and isolation are performed since an estimate of the fault affecting the system is available.

\section{FAult tOlERANT CONTROLLER DESIGN}

Along of this study, we assume that:

1) The faults and the system states are observable from the output.

2) The nonlinear functions depend only on the measurable variables $y(t)$ or $u(t)$.

3) The considered fault functions are differentiable.

\section{A. FTC for exponential faults}

Assume that the faults affecting the system are modeled by exponential function $\left(f_{i}(t) \equiv e^{\alpha_{i} t+\beta_{i}}\right.$, with $\alpha_{i}, \beta_{i} \in \mathbb{R}$, $i=1, \ldots, q$. In the next, we consider that $\alpha_{i}=\alpha_{0, i}+\Delta \alpha_{i}$, which allows defining a set of exponential functions, $\alpha_{0, i}$ and $\Delta \alpha_{i}$ representing respectively the nominal and the uncertain parts of $\alpha_{i}$. Let us define $\alpha=\operatorname{diag}\left(\alpha_{1}, \ldots, \alpha_{q}\right)$, $\alpha_{0}=\operatorname{diag}\left(\alpha_{0,1}, \ldots, \alpha_{0, q}\right)$, and $\Delta \alpha=\operatorname{diag}\left(\Delta \alpha_{1}, \ldots, \Delta \alpha_{q}\right)$. The uncertain part can be bounded as:

$$
(\Delta \alpha)^{T} \Delta \alpha \leq v
$$

where $v \in \mathbb{R}^{q \times q}$ is a known diagonal positive definite matrix.

Let us respectively define the state and fault estimation errors as: $e_{s}(t)=x_{f}(t)-\hat{x}_{f}(t)$ and $e_{d}(t)=f(t)-\hat{f}(t)$. Let us also define the state tracking error $e_{p}(t)=x(t)-x_{f}(t)$, the output error $e_{y}(t)=y_{f}(t)-\hat{y}_{f}(t)$ and the error between the nominal and FTC control signals $e_{u}(t)=u(t)-u_{f}(t)$.

According to the above notations, the dynamics of $e_{p}(t)$ and $e_{S}(t)$ are given by:

$$
\begin{aligned}
& \dot{e}_{p}(t)=A_{\mu} e_{p}(t)+B_{\mu} e_{u}(t)-G_{\mu} f(t) \\
& \dot{e}_{S}(t)=A_{\mu} e_{s}(t)+G_{\mu} e_{d}(t)-H_{\mu}^{1} e_{y}(t)
\end{aligned}
$$

Since $\dot{f}(t)=\alpha f(t)$, the dynamics of the fault estimation error is given by:

$$
\dot{e}_{d}(t)=\alpha f(t)-H_{\mu}^{2} e_{y}(t)+H_{\mu}^{3} \hat{f}(t)
$$

By adding and subtracting $H_{\mu}^{3} f(t)$ in (12), one can obtain:

$$
\dot{e}_{d}(t)=-H_{\mu}^{2} e_{y}(t)-H_{\mu}^{3} e_{d}(t)+\left(\alpha+H_{\mu}^{3}\right) f(t)
$$


The substitution of the expression of $e_{y}(t)$ in (11) and (13) and the expression of $e_{u}(t)$ in (10), leads to introducing multiplications between the system matrices and the ones of the observer and the controller. This coupling leads to conservative results. A way to overcome this problem is to introduce a "virtual dynamics" in the $e_{y}(t)$ and $e_{u}(t)$ equations [21][22][23]. This craftiness allows decoupling the system, observer and FTC controller matrices in the expressions of the error dynamics. Hence, $e_{y}(t)$ and $e_{u}(t)$ can be rewritten as:

$$
0 \dot{e}_{y}(t)=-e_{y}(t)+C_{\mu} e_{s}(t)+W_{\mu} e_{d}(t)
$$

where $0 \in \mathbb{R}^{p \times p}$ is a zero matrix.

Adding and subtracting $K_{\mu} x_{f}(t)$ and $K_{\mu}^{f} f(t)$ in $e_{u}(t)$, one can obtain:

$$
0 \dot{e}_{u}(t)=-K_{\mu}^{f} f(t)+K_{\mu}^{f} e_{d}(t)+K_{\mu} e_{p}(t)+K_{\mu} e_{s}(t)+e_{u}(t)
$$

where $0 \in \mathbb{R}^{m \times m}$ is a zero matrix. The concatenation of (10), (11), (13), (14) and (15) leads to the following descriptor representation:

$$
E \dot{\tilde{e}}(t)=\tilde{A}_{\mu} \tilde{e}(t)+\tilde{B}_{\mu} f(t)
$$

where $E=\operatorname{diag}\left(I_{n} I_{n} I_{q} 0_{p} 0_{m}\right)$,

$$
\begin{gathered}
\tilde{e}^{T}(t)=\left(e_{p}^{T}(t) e_{s}^{T}(t) e_{d}^{T}(t) e_{y}^{T}(t) e_{u}^{T}(t)\right), \\
\tilde{A}_{\mu}=\left(\begin{array}{ccccc}
A_{\mu} & 0 & 0 & 0 & B_{\mu} \\
0 & A_{\mu} & G_{\mu} & -H_{\mu}^{1} & 0 \\
0 & 0 & -H_{\mu}^{3} & -H_{\mu}^{2} & 0 \\
0 & C_{\mu} & W_{\mu} & -I & 0 \\
-K_{\mu} & -K_{\mu} & -K_{\mu}^{f} & 0 & -I
\end{array}\right)^{T} \\
\text { and } \tilde{B}_{\mu}^{T}=\left(\begin{array}{ccccc}
-G_{\mu}^{T} & 0 & \left(\alpha+H_{\mu}^{3}\right)^{T} & 0 & \left(K_{\mu}^{f}\right)^{T}
\end{array}\right) .
\end{gathered}
$$

From the structure of the matrices $E$ and $\tilde{A}_{\mu}$, we remark that the descriptor (16) is impulse-free. The main interest of the descriptor approach is to avoid products of system, observer and controller gains in the LMI, and thus obtain more tractable and less restrictive conditions.

The main provided results are summarized in the following theorem 1 .

Theorem 1: The system (16) that describes the different estimation errors is stable and the $\mathscr{L}_{2}$-gain from the faults to the state tracking error, the state and fault estimation errors is bounded by $\sqrt{\gamma}$, if there exists matrices $P_{1}=P_{1}^{T} \geqslant 0$, $P_{7}=P_{7}^{T} \geqslant 0, P_{13}=P_{13}^{T} \geqslant 0, P_{16}, P_{17}, P_{18}, P_{19}, P_{25}, F_{i}, R_{i}$, $S_{i}, Q_{i}$ and $M_{i}$ and positive scalars $\bar{\gamma}$ and $\tau$ such that the following LMI are verified, for $i=1, \ldots, r$ :

$$
\left(\begin{array}{ccccccc}
\Upsilon_{i}^{1,1} & * & * & * & * & * & 0 \\
C_{i}^{T} P_{16} & \Upsilon_{i}^{2,2} & * & * & * & 0 & 0 \\
W_{i}^{T} P_{16} & \Upsilon_{i}^{3,2} & \Upsilon_{i}^{3,3} & * & * & * & * \\
-P_{16} & \Upsilon_{i}^{4,2} & \Upsilon_{i}^{4,3} & \Upsilon^{4,4} & 0 & 0 & 0 \\
\Upsilon_{i}^{5,1} & -F_{i} & -Q_{i} & 0 & \Upsilon^{5,5} & * & 0 \\
-G_{i}^{T} P_{1} & 0 & \Upsilon_{i}^{6,3} & 0 & Q_{i}^{T} & (\tau-\bar{\gamma}) I & 0 \\
0 & 0 & P_{13} & 0 & 0 & 0 & -\tau \nu I
\end{array}\right)<0
$$

where

$$
\begin{aligned}
& \Upsilon_{i}^{1,1}=P_{1} A_{i}+A_{i}^{T} P_{1}+I, \\
& \Upsilon_{i}^{2,2}=P_{7} A_{i}+A_{i}^{T} P_{7}+P_{17}^{T} C_{i}+C_{i}^{T} P_{17}+I, \\
& \Upsilon_{i}^{3,2}=P_{18}^{T} C_{i}+G_{i}^{T} P_{7}+W_{i}^{T} P_{17}, \\
& \Upsilon_{i}^{3,3}=-M_{i}^{T}-M_{i}+P_{18}^{T} W_{i}+W_{i}^{T} P_{18}+I, \\
& \Upsilon_{i}^{r^{4,2}}=P_{19}^{T} C_{i}-P_{17}-S_{i}, \\
& \Upsilon_{i}^{r^{4,3}}=P_{19}^{T} W_{i}-P_{18}-R_{i}^{T}, \\
& \Upsilon^{4,4}=-P_{19}-P_{19}^{T}, \Upsilon_{i}^{5,1}=-F_{i}+B_{i}^{T} P_{1}, \\
& \Upsilon^{5,5}=-P_{25}-P_{25}^{T} \text { and } \\
& \Upsilon_{i}^{6,3}=\alpha_{0} P_{13}+M_{i} .
\end{aligned}
$$

Proof. : To study the stability of (16), we consider the following quadratic Lyapunov function candidate:

$$
V\left(e_{p}(t), e_{s}(t), e_{d}(t)\right)=\tilde{e}^{T}(t) E P \tilde{e}(t)
$$

with

$$
E P=P^{T} E \geqslant 0
$$

The choice of the Lyapunov function (18) will ensure the stability of the tracking error and of the state and fault estimations. To attenuate the fault effect on the error dynamics, one considers the $\mathscr{L}_{2}$ constraint [24] given by:

$$
\int_{0}^{t_{f}} \tilde{e}^{T}(t) E \tilde{e}(t) \leqslant \gamma^{2} \int_{0}^{t_{f}} f^{T}(t) f(t)
$$

where $t_{f}$ and $\gamma$ represent respectively the final time and the attenuation level. The tracking error $e_{p}(t)$, state $e_{s}(t)$ and fault $e_{d}(t)$ estimation errors must therefore satisfy the following inequality:

$\dot{\tilde{e}}^{T}(t) E P \tilde{e}(t)+\tilde{e}^{T}(t) E P \dot{\tilde{e}}(t)+\tilde{e}^{T}(t) E \tilde{e}(t)-\gamma^{2} f^{T}(t) f(t)<0$

Considering (19) and substituting (16) in (21), one can obtain:

$$
\left(\begin{array}{c}
\tilde{e}^{T}(t) \\
f^{T}(t)
\end{array}\right)^{T}\left(\begin{array}{cc}
\tilde{A}_{\mu}^{T} P+P^{T} \tilde{A}_{\mu}+E & * \\
\tilde{B}_{\mu}^{T} P & -\gamma^{2}
\end{array}\right)\left(\begin{array}{c}
\tilde{e}(t) \\
f(t)
\end{array}\right)<0
$$

The inequality (22) is fulfilled if:

$$
\left(\begin{array}{cc}
\tilde{A}_{\mu}^{T} P+P^{T} \tilde{A}_{\mu}+E & * \\
\tilde{B}_{\mu}^{T} P & -\gamma^{2}
\end{array}\right)<0
$$

The structure of the Lyapunov matrix $P$ is chosen as follows:

$$
P=\left(\begin{array}{ccccc}
P_{1} & 0 & 0 & 0 & 0 \\
0 & P_{7} & 0 & 0 & 0 \\
0 & 0 & P_{13} & 0 & 0 \\
P_{16} & P_{17} & P_{18} & P_{19} & 0 \\
0 & 0 & 0 & 0 & P_{25}
\end{array}\right)
$$

Clearly, this structure is not the general one but it limits the coupling between the unknown submatrices to be determined and allows the convergence conditions to be expressed using LMI. According to (19), this latter imposes that $P_{1}=P_{1}^{T} \geqslant 0$, $P_{7}=P_{7}^{T} \geqslant 0, P_{13}=P_{13}^{T} \geqslant 0$ and $P_{16}, P_{17}, P_{18}, P_{19} P_{25}$ are free slack matrices with appropriate dimensions. Considering 
(24) and the matrices defined in (16), the mathematical development of (23) leads to:

$$
\Xi_{\mu}+\Gamma^{T} \Omega+\Omega^{T} \Gamma<0
$$

where $\Gamma=\left(\begin{array}{llllll}0 & 0 & 0 & 0 & 0 & \Delta \alpha\end{array}\right)$,

$\Omega=\left(\begin{array}{cccccc}0 & 0 & P_{13} & 0 & 0 & 0\end{array}\right)$ and

$$
\Xi_{\mu}=\left(\begin{array}{cccccc}
\Psi_{\mu}^{1,1} & * & * & * & * & * \\
C_{\mu}^{T} P_{16} & \Psi_{\mu}^{2,2} & * & * & * & 0 \\
W_{\mu}^{T} P_{16} & \Psi_{\mu}^{3,2} & \Psi_{\mu}^{3,3} & * & * & * \\
-P_{16} & \Psi_{\mu}^{4,2} & \Psi_{\mu}^{4,3} & \Psi^{4,4} & 0 & 0 \\
\Psi_{\mu}^{5,1} & -P_{25}^{T} K_{\mu} & -P_{25}^{T} K_{\mu}^{f} & 0 & \Psi^{5,5} & * \\
-G_{\mu}^{T} P_{1} & 0 & \Psi_{\mu}^{6,3} & 0 & \Psi_{\mu}^{6,5} & -\gamma^{2} I
\end{array}\right)
$$

with

$$
\begin{array}{ll}
\Psi_{\mu}^{1,1}=P_{1} A_{\mu}+A_{\mu}^{T} P_{1}+I, \\
\Psi_{\mu}^{2,2}=P_{7} A_{\mu}+A_{\mu}^{T} P_{7}+P_{17}^{T} C_{\mu}+C_{\mu}^{T} P_{17}+I, \\
\Psi_{\mu}^{3,2}=P_{18}^{T} C_{\mu}+G_{\mu}^{T} P_{7}+W_{\mu}^{T} P_{17}, \\
\Psi_{\mu}^{3,3}=-P_{13}^{T} H_{\mu}^{3}-\left(H_{\mu}^{3}\right)^{T} P_{13}+P_{18}^{T} W_{\mu}+W_{\mu}^{T} P_{18}+I, \\
\Psi_{\mu}^{4,2}=P_{19}^{T} C_{\mu}-P_{17}-\left(H_{\mu}^{1}\right)^{T} P_{7}, \\
\Psi_{\mu}^{4,3}=P_{19}^{T} W_{\mu}-P_{18}-\left(H_{\mu}^{2}\right)^{T} P_{13}, & \Psi^{4,4}=-P_{19}-P_{19}^{T}, \\
\Psi_{\mu}^{5,1}=-P_{25}^{T} K_{\mu}+B_{\mu}^{T} P_{1}, & \Psi^{5,5}=-P_{25}-P_{25}^{T}, \\
\Psi_{\mu}^{6,3}=\alpha_{0} P_{13}+\left(H_{\mu}^{3}\right)^{T} P_{13}, & \Psi_{\mu}^{6,5}=\left(K_{\mu}^{f}\right)^{T} P_{25} .
\end{array}
$$

Applying lemma 1 on (25), then considering (9), (25) can be bounded as:

$$
\Xi_{\mu}+\tau \Lambda^{T} \Lambda+\tau^{-1} \Omega^{T} \Omega<0
$$

with $\Lambda=\left(\begin{array}{llllll}0 & 0 & 0 & 0 & 0 & v\end{array}\right)$. To provide LMI conditions, we consider the following bijective changes of variables:

$$
\begin{aligned}
& \left(H_{\mu}^{3}\right)^{T} P_{13}=M_{\mu}, \quad P_{25}^{T} K_{\mu}=F_{\mu}, \quad P_{13}^{T} H_{\mu}^{2}=R_{\mu}, \\
& \left(H_{\mu}^{1}\right)^{T} P_{7}=S_{\mu}, \quad P_{25}^{T} K_{\mu}^{f}=Q_{\mu}, \quad \bar{\gamma}=\gamma^{2},
\end{aligned}
$$

then applying Schur complement [24] on the term $\tau^{-1} \Omega^{T} \Omega$, thus, the sufficient LMI conditions proposed in theorem 1 hold.

\section{B. FTC for first order polynomial faults}

Let us now consider that the fault affecting the system are modeled by the first order plynomial given by:

$$
f_{i}(t)=\lambda_{i} t+\delta_{i}
$$

where $\lambda_{i} \in \mathbb{R}$ and $\delta_{i} \in \mathbb{R}, i=1, \ldots, q$. As well as for exponential function, $\lambda=\lambda_{0}+\Delta \lambda$, with $\Delta \lambda$ verifying:

$$
(\Delta \lambda)^{T} \Delta \lambda \leq v
$$

where $v \in \mathbb{R}^{q \times q}$ is a known diagonal positive definite matrix.

According to (13), the fault estimation error dynamics is given by:

$$
\dot{e}_{d}(t)=-H_{\mu}^{2} e_{y}(t)-H_{\mu}^{3} e_{d}(t)+\left(H_{\mu}^{3}\right) f(t)+\lambda
$$

The combination of (10), (11), (29), (14) and (15) leads to the following descriptor representation:

$$
E \dot{\tilde{e}}(t)=\tilde{A}_{\mu} \tilde{e}(t)+\bar{B}_{\mu} f(t)+N
$$

where the matrices $E$ and $\tilde{A}_{\mu}$ are defined in equation (16) and $N^{T}=\left(\begin{array}{ccccc}0 & 0 & \lambda^{T} & 0 & 0\end{array}\right)$, $\bar{B}_{\mu}^{T}=\left(\begin{array}{lllll}-G_{\mu}^{T} & 0 & \left(H_{\mu}^{3}\right)^{T} & 0 & \left(K_{\mu}^{f}\right)^{T}\end{array}\right)$.

As well as for the case of exponential faults, the obtained desciptor (30) is impulse-free.

The main provided results are summarized in the following theorem 2.

Theorem 2: The system (30) that describes the different estimation errors is stable and the $\mathscr{L}_{2}$-gain from the faults to the state tracking error, the state and fault estimation errors is bounded by $\sqrt{\gamma}$, if there exists matrices $P_{1}=P_{1}^{T} \geqslant 0$, $P_{7}=P_{7}^{T} \geqslant 0, P_{13}=P_{13}^{T} \geqslant 0, P_{16}, P_{17}, P_{18}, P_{19}, P_{25}, F_{i}, R_{i}$, $S_{i}, Q_{i}$ and $M_{i}$ and positive scalars $\bar{\gamma}, \rho$ and $\sigma$ such that the following LMI are verified: for $i=1, \ldots, r$

$$
\left(\begin{array}{cccccccc}
\Phi_{i}^{1,1} & * & * & * & * & * & 0 & 0 \\
\Phi_{i}^{2,1} & \Phi_{i}^{2,2} & * & * & * & 0 & 0 & 0 \\
\Phi_{i}^{3,1} & \Phi_{i}^{3,2} & \Phi_{i}^{3,3} & * & * & * & * & 0 \\
-P_{16} & \Phi_{i}^{4,2} & \Phi_{i}^{4,3} & \Phi^{4,4} & 0 & 0 & 0 & 0 \\
\Phi_{i}^{5,1} & -F_{i} & -Q_{i} & 0 & \Phi^{5,5} & * & 0 & 0 \\
\Phi_{i}^{6,1} & 0 & M_{i} & 0 & Q_{i}^{T} & -\overline{\gamma I} & 0 & 0 \\
0 & 0 & \Phi_{i}^{7,3} & 0 & 0 & 0 & \Phi^{7,7} & * \\
0 & 0 & 0 & 0 & 0 & 0 & P_{13} & -\sigma I
\end{array}\right)<0
$$

where

$$
\begin{array}{lll}
\Phi_{i}^{1,1}=\Upsilon_{i}^{1,1}+\rho I, & \Phi_{i}^{2,1}=C_{i}^{T} P_{16}, & \Phi_{i}^{2,2}=\Upsilon_{i}^{2,2}+\rho I, \\
\Phi_{i}^{3,1}=W_{i}^{T} P_{16}, & \Phi_{i}^{3,2}=\Upsilon_{i}^{3,2}, & \Phi_{i}^{3,3}=\Upsilon_{i}^{3,3}+(\sigma v+\rho) I, \\
\Phi_{i}^{4,2}=\Upsilon_{i}^{4,2}, & \Phi_{i}^{4,3}=\Upsilon_{i}^{4,3}, & \Phi_{i}^{4,4}=\Upsilon_{i}^{4,4}+\rho I, \\
\Phi_{i}^{5,1}=\Upsilon_{i}^{5,1}, & \Phi_{i}^{5,5}=\Upsilon_{i}^{5,5}+\rho I, & \Phi_{i}^{6,1}=-G_{i}^{T} P_{1}, \\
\Phi^{7,3}=\lambda_{0} P_{13}, & \Phi^{7,7}=-\varepsilon \rho I . &
\end{array}
$$

Proof. Considering (18), (19), (30) and following the same path as for the proof of theorem 1 ((18)-(22)), one can obtain:

$$
\left(\begin{array}{c}
\tilde{e}^{T} \\
f^{T} \\
I
\end{array}\right)^{T}\left(\begin{array}{ccc}
\tilde{A}_{\mu}^{T} P+P^{T} \tilde{A}_{\mu}+E & * & * \\
\bar{B}_{\mu}^{T} P & -\gamma^{2} I & 0 \\
N^{T} P & 0 & 0
\end{array}\right)\left(\begin{array}{c}
\tilde{e} \\
f \\
I
\end{array}\right)<0
$$

To ensure the asymptotic convergence to a ball of radius $\varepsilon$ of the error dynamics, we consider the following constraint:

$$
\|\tilde{e}\|_{2}^{2} \geq \varepsilon I
$$

with $\varepsilon$ is a known small positive scalar. The inequality (33) is equivalent to:

$$
\left(\begin{array}{c}
\tilde{e}^{T} \\
f^{T} \\
I
\end{array}\right)^{T}\left(\begin{array}{ccc}
I & 0 & 0 \\
0 & 0 & 0 \\
0 & 0 & -\varepsilon I
\end{array}\right)\left(\begin{array}{c}
\tilde{e} \\
f \\
I
\end{array}\right) \geq 0
$$

Applying S-procedure lemma 2 [24] on (33) and (34), one can obtain:

$$
\left(\begin{array}{c}
\tilde{e}^{T} \\
f^{T} \\
I
\end{array}\right)^{T}\left(\begin{array}{ccc}
\Psi_{\mu} & * & * \\
\bar{B}_{\mu}^{T} P & -\gamma^{2} I & 0 \\
N^{T} P & 0 & -\varepsilon \rho I
\end{array}\right)\left(\begin{array}{c}
\tilde{e} \\
f \\
I
\end{array}\right)<0
$$


where $\Psi_{\mu}=\tilde{A}_{\mu}^{T} P+P^{T} \tilde{A}_{\mu}+E+\rho I$.

Considering the matrix $N$ defined in (30) and $P$, (35) fulfilled if:

$$
\chi_{\mu}+\Sigma^{T} \phi+\phi^{T} \Sigma<0
$$

where $\Sigma=\left(\begin{array}{ccccccc}0 & 0 & 0 & 0 & 0 & 0 & \Delta \lambda\end{array}\right)$, $\phi=\left(\begin{array}{lllllll}0 & 0 & P_{13} & 0 & 0 & 0 & 0\end{array}\right)$ and

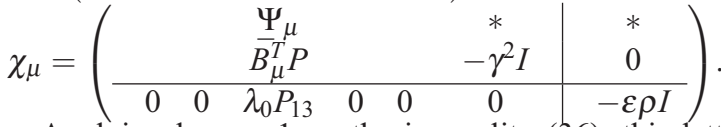

Applying lemma 1 on the inequality (36), this latter c: be written as:

$$
\chi_{\mu}+\sigma \Sigma^{T} \Sigma+\sigma^{-1} \phi^{T} \phi<0
$$

Following the same steps of the proof of theorem 1 from (23) to the end, then applying the Schur complement [24] on the term $\sigma^{-1} \phi^{T} \phi$, thus, the sufficient LMI conditions proposed in theorem 2 hold

\section{Simulation example}

In this section, the effectiveness and the applicability of the proposed approach are illustrated. An academic example is considered since the main purpose of this paper is theoretical. Let us consider the following Takagi-Sugeno model:

$$
\left\{\begin{array}{l}
\dot{x}(t)=\sum_{i=1}^{2} \mu_{i}(\xi(u(t)))\left(A_{i} x_{f}(t)+B_{i} u_{f}(t)+G_{i} f(t)\right) \\
y(t)=\sum_{i=1}^{2} \mu_{i}(\xi(u(t)))\left(C_{i} x_{f}(t)+D_{i} u_{f}(t)+W_{i} f(t)\right)
\end{array}\right.
$$

with $A_{1}=\left(\begin{array}{ccc}-5 & 1 & -3 \\ 1 & -3 & 2 \\ 1 & 1 & -4\end{array}\right), A_{2}=\left(\begin{array}{ccc}-3 & 1 & 1 \\ 0.5 & -3 & 2 \\ 0.5 & 1 & -5\end{array}\right)$, $B_{1}=\left(\begin{array}{l}5 \\ 3 \\ 2\end{array}\right), B_{2}=\left(\begin{array}{l}5 \\ 4 \\ 5\end{array}\right), G_{1}=\left(\begin{array}{l}5 \\ 5 \\ 0\end{array}\right), G_{2}=\left(\begin{array}{l}5 \\ 5 \\ 5\end{array}\right)$, $W_{1}=\left(\begin{array}{c}-0.5 \\ 0.5\end{array}\right), W_{2}=\left(\begin{array}{c}-1 \\ 0.5\end{array}\right), C_{1}=\left(\begin{array}{ccc}0.5 & 0.5 & 0 \\ 0 & 0.5 & 0\end{array}\right)$, $C_{2}=\left(\begin{array}{ccc}1 & 0.5 & 0 \\ 0.5 & 1 & 0\end{array}\right), D_{1}=\left(\begin{array}{c}-0.8 \\ 0\end{array}\right), D_{2}=\left(\begin{array}{c}-0.8 \\ -0.5\end{array}\right)$, $\mu_{1}(u(t))=\frac{1-\tanh (0.5-u(t))}{2}$ and $\mu_{2}(u(t))=1-\mu_{1}(u(t))$. The nominal input signal is $u(t)=\sin (\cos (0.5 t) t)$. The LMI conditions given in theorems 1 and 2 have been solved by using Matlab LMI Toolbox.

\section{A. Case of exponential fault}

Let us consider the following exponential fault affecting the system behavior occurs at $8 \mathrm{sec} \leq t \leq 11 \mathrm{sec}$ :

$$
f(t)=e^{1.15 t-14}
$$

The controller and the observer are synthesized for $\alpha_{0}=1$ and $\Delta \alpha=0.2$. Thus, the simulation results are illustrated by the figures 2 and 3 .

From these latter, one can see that the synthesized observer and FTC controller showed their effectiveness, since the fault and the system states are estimated and the tracking between the faulty system states and the reference model ones is ensured.
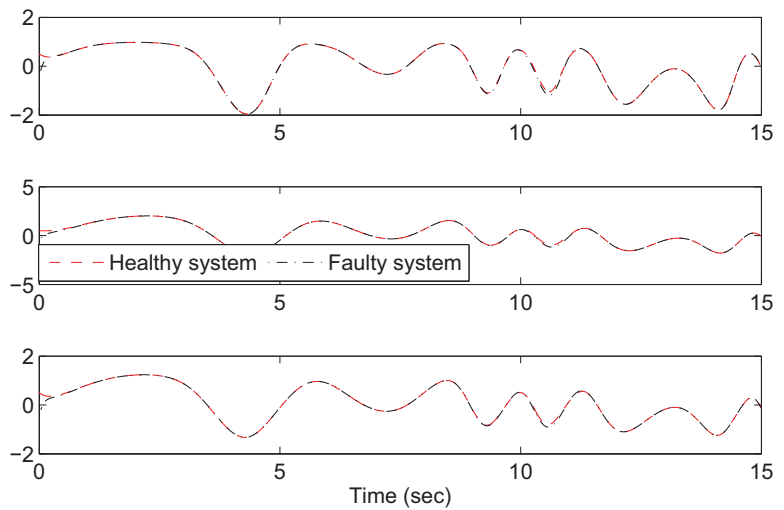

Fig. 2. Reference model states vs. faulty system ones with FTC
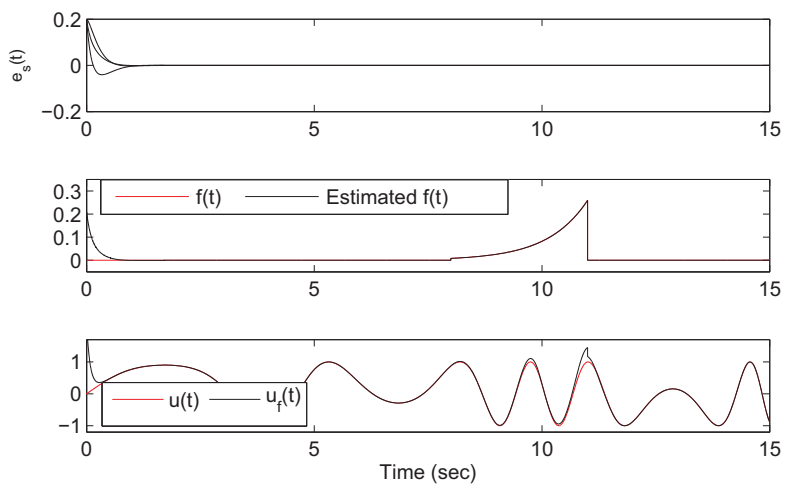

Fig. 3. State estimation errors, fault and its estimation, nominal and FTC control inputs

\section{B. Case of first order polynomial fault}

The fault affecting the system occurs at $9 \mathrm{sec} \leq t \leq 12.5 \mathrm{sec}$ and is given by the following first order polynomial:

$$
f(t)=0.2 t-1
$$

Note that the FTC controller and observer gains are computed for $\varepsilon=10^{-3}, \lambda_{0}=0.11$ and $\Delta \lambda=0.25$. Thus, the simulation results are illustrated by the following figures.

It can be seen that the synthesized FTC controller compensates the fault and ensures the tracking between the reference and the faulty system states, even if the parameter of the occuring fault is not perfectly known for observer and controller designs.

\section{CONCLUSiOn}

In this paper, fault tolerant tracking controller design approaches for continuous Takagi-Sugeno models are proposed. They concern the case when the fault affecting the system behavior are time varying. In this study, the considered faults are modeled by exponential function or first order polynomial. The provided conditions are easily formulated in term of LMI using the well known descriptor redundancy property. This latter allows introducing free slack variables in the Lyapunov function candidate leading to 

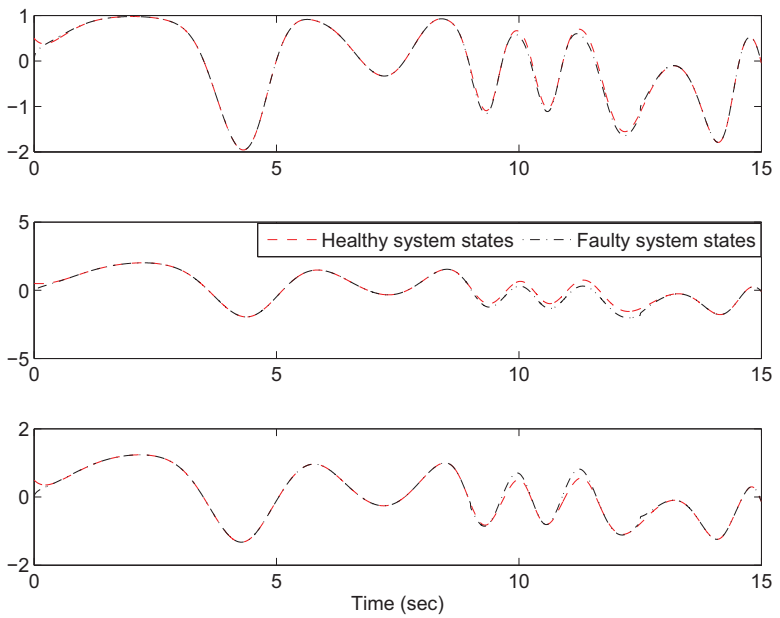

Fig. 4. Reference model states vs. faulty system ones with FTC
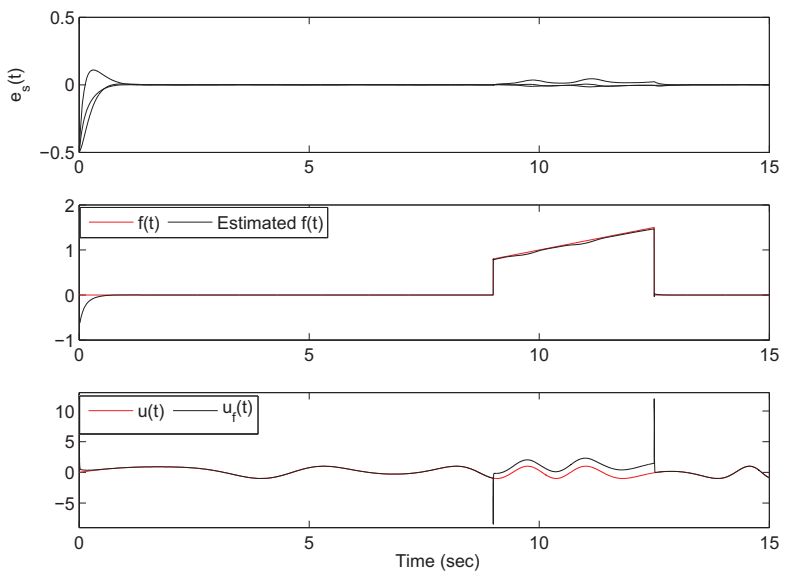

Fig. 5. State estimation errors, fault and its estimation, nominal and FTC control inputs

less conservative LMI conditions. The effectiveness of the proposed approaches are illustrated with an example. Only an academic example was considered, since the present paper provides preliminary theoretical results, more efforts will be made to improve applicability to real processes.

\section{REFERENCES}

[1] Z. Gao and P.J. Antsaklis, Reconfigurable control system design via perfect model following, International Journal of Control, 56(4):,783798, 1992

[2] G. Liu and R. Patton, Eigenstructure Assignment for control systems design, John Wiley \& Sons, New York, 1998.

[3] H. Noura, D. Sauter, F. Hamelin and D. Theilliol, Fault-tolerant control in dynamic systems: Application to a winding machine, IEEE Control Systems Magazine, 20(1):33-49, 2000

[4] M.M. Mufeed, J. Jiang and Z. Zhang, Active fault tolerant control systems, Stochastic Analysis and Synthesis, Springer 2003.

[5] B. Marx, D. Koenig and D. Georges, A Robust fault tolerant control for descriptor systems, IEEE Transactions on Automatic Control, 49(10):1869-1875, 2004

[6] M. Staroswiecki, Fault tolerant control: the pseudo-inverse method revised, In 16th IFAC World Congress, Seoul, Korea, 2005.
[7] Y. Zhang and J. Jiang, Bibliographical review on reconfigurable faulttolerant control systems, Annual Reviews in Control, 32(2):229-252, 2008.

[8] Z. Zuo, D. W.C. Ho, and Y. Wang, Fault tolerant control for singular systems with actuator saturation and nonlinear perturbation, Automatica, 46(3):569-576, 2010.

[9] Y. Shtessel, J. Buffington and S. Banda, Tailless aircraft flight control using multiple time scale reconfigurable sliding modes, IEEE Trans actions on Control Systems Technology, 10(2):288-296, 2002.

[10] R. A. Hess and S. R. Wells, Sliding mode control applied to reconfigurable flight control design, Journal of Guidance Control and Dynamics, 26(3):452-462, 2003.

[11] D. Theilliol, H. Noura, and J.C. Ponsart, Fault diagnosis and accommodation of a three-tank system based on analytical redundancy, ISA Transactions, 41(3):365-382, 2002.

[12] T. Takagi and M. Sugeno, Fuzzy identification of systems and its application to modeling and control, IEEE Transactions on System Man and Cybernetics, 15(1):116-132, 1985.

[13] K. Tanaka and H.O. Wang, Fuzzy control systems design and analysis. a linear matrix inequality approach, John Wiley \& Sons, New York, 2001.

[14] G. Feng, A survey on analysis and design of model-based fuzzy control systems, IEEE Transactions on Fuzzy Systems, 14(5):676-697, 2006.

[15] T. M. Guerra, A. Kruszewski and J. Lauber, Discrete Takagi-Sugeno models for control: Where are we?, Annual Reviews in Control, 33(1):37-47, 2009

[16] A. Sala, On the conservativeness of fuzzy and fuzzy-polynomial control of nonlinear systems, Annual Reviews in Control, 33(1):48 $58,2009$.

[17] T. Bouarar, B. Marx, D. Maquin and J. Ragot, Trajectory tracking fault tolerant controller design for Takagi-Sugeno systems subject to actuator faults, In International Conference on Communications, Computing and Control Applications, Hammamet, Tunisia, 2011

[18] D. Ichalal, B. Marx, J. Ragot and D. Maquin, Fault tolerant control for Takagi-Sugeno systems with unmeasuable premise variables by trajectory tracking, In IEEE International Symposium on Industrial Electronics, pp. 2097-2102, Bari, Italy, 2010.

[19] D. Ichalal, B. Marx, J. Ragot and D. Maquin, Observer based actuator fault tolerant control in nonlinear Takagi-Sugeno fuzzy systems: LMI approach, In 18th IEEE Mediterranean Conference on Control and Automation, pp. 1278-1283, Marrakech, Morocco, 2010.

[20] K. Zhou and P.P. Khargonekar, Robust stabilization of linear systems with norm-bounded time-varying uncertainty, Systems and Control Letters, 10(1):17-20, 1988.

[21] K. Guelton, T. Bouarar and N. Manamanni, Robust dynamic output feedback fuzzy Lyapunov stabilization of Takagi-Sugeno systems - A descriptor redundancy approach, Fuzzy Sets and Systems, 160(19):2796-2811, 2009.

[22] T. Bouarar, K. Guelton and N. Manamanni, Static Output Feedback Controller Design for Takagi-Sugeno Systems - A Fuzzy Lyapunov LMI Approach, In 48th IEEE Conference on Decision and Control, pp.4150-4155, Shanghai, China, 2009

[23] T. Bouarar, K. Guelton and N. Manamanni, Robust Fuzzy Lyapunov Stabilization for Uncertain and Disturbed Takagi-Sugeno Descriptors, ISA Transactions, 49(4):447-461, 2010.

[24] S. Boyd, L. El Ghaoui, E. Féron, and V. Balakrishnan, Linear Matrix Inequalities, System and Control Theory, Studies in Applied Mathematics, Philadelphia, PA: SIAM, 1994. 